\title{
ATUAÇÃO FISIOTERAPÊUTICA NO MAL DE ALZHEIMER: UMA REVISÃO DA LITERATURA
}

\section{ARTIGO DE REVISÃo}

OLIVEIRA, Tâmara Lourdes da Silva ${ }^{1}$

OLIVEIRA, Tâmara Lourdes da Silva. Atuação fisioterapêutica no mal de Alzheimer: uma revisão da literatura. Revista Científica Multidisciplinar Núcleo do Conhecimento. Ano. 06, Ed. 12, Vol. 08, pp. 168-175. Dezembro de 2021. ISSN: 24480959, Link de acesso: https://www.nucleodoconhecimento.com.br/saude/fisioterapeutica

\section{RESUMO}

Restaurar funções como equilíbrio, força e coordenação motora, são alguns dos benefícios que a fisioterapia traz para o idoso, principalmente para aqueles que já possuem algum quadro patológico em curso. Geralmente estas doenças causam um déficit nas funções básicas, como na locomoção e coordenação motora. A doença de Alzheimer, em estágios mais avançados, deixa o idoso debilitado, acamado e com várias disfunções, como por exemplo: dificuldade para deambular normalmente. Portanto, a fisioterapia atua de modo a evitar e/ou retardar as adversidades causadas pela doença de Alzheimer. O objetivo do estudo foi verificar por meio da literatura a atuação fisioterapêutica no mal de Alzheimer. Para isto, adotou-se como metodologia a revisão da literatura, construída por meio de consulta às bases de dados e outros trabalhos relacionados ao tema, nos meses de julho de 2020 a outubro de 2021, respondendo à questão norteadora: Quais as condutas fisioterapêuticas descritas na literatura para os pacientes com doença de Alzheimer? Foram selecionados 13 trabalhos que explicitaram a importância da fisioterapia diante do tema exposto, sendo pontuada sua atuação no início da doença, desde a educação em saúde e orientações

\footnotetext{
1 Pós-graduada em fisioterapia em gerontologia. (centro universitário estácio de sá) Graduada em fisioterapia. (universidade paulista-unip). ORCID: 0000-000338496550
} 
aos familiares. Pode-se concluir que, a atuação fisioterapêutica no mal de Alzheimer é benéfica aos pacientes portadores da afecção neurodegenerativa, não só do ponto de vista físico e motor, mas, também, cognitivo e respiratório, e que esta possui meios para reabilitar e retardar perdas funcionais, evitar encurtamentos musculares, promover a independência funcional, melhorando assim a qualidade de vida.

Palavras-Chaves: Atuação, fisioterapêutica, mal de Alzheimer.

\section{INTRODUÇÃO}

Um dos maiores avanços da humanidade foi a prolongação da vida, isso fica claro nas estatísticas que apontam elevação no número de pessoas com 60 anos ou mais, especialmente, com prevalência do sexo feminino. (SOUSA et al., 2018). Porém, é importante envelhecer de forma saudável, agregando qualidade de vida a esse processo fisiológico que é normal e esperado. (VERAS e OLIVEIRA, 2018).

O envelhecimento fisiológico compromete todos os sistemas do corpo humano. No sistema músculo esquelético ocorre perda de massa muscular e de células responsáveis pela síntese dos componentes orgânicos da matriz óssea, ocasionado quedas e consequentemente fraturas ósseas. (MANSO et al., 2019). No sistema nervoso, a habilidade em processar, realizar sinais visuais e de propriocepção são perdidas, causando alterações no equilíbrio corporal e nos reflexos. (BUSHATSKY et al., 2019).

A doença de Alzheimer é uma patologia neurodegenerativa e progressiva. Possui maior incidência com o avançar da idade, levando a uma perda de memória e funções cognitivas que interferem nas atividades de vida diária e nas atividades instrumentais. Sendo fisioterapia benéfica em todas as fases doença (SANTANA et al., 2019; SILVA; SANTANA; RODRIGUES, 2019).

Através de técnicas fisioterapêuticas é possível melhorar a qualidade de vida do paciente com a doença de Alzheimer, retardando perdas funcionais e reduzindo complicações devido a imobilizações. Ainda é possível a melhora do equilíbrio corporal, prevenir quedas, melhora da marcha, evitar encurtamentos musculares, 
perda de massa muscular e força muscular, diminuição de tônus, prevenir deformidades, promovendo assim a independência funcional. (LIMA et al., 2016; MEDEIROS et al., 2015; SOARES, 2019).

Portanto, o presente artigo, visa responder: Quais as condutas fisioterapêuticas descritas na literatura para os pacientes com doença de Alzheimer? Tendo como objetivo demonstrar, através de uma revisão bibliográfica, a atuação fisioterapêutica no mal de Alzheimer, suas condutas e benefícios para os pacientes portadores desta afecção neurodegenerativa.

\section{METODOLOGIA}

Trata-se de uma revisão bibliográfica da literatura, realizada nas bases de dados: Google acadêmico, Revista Brasileira de epidemiológica v.21, Revista Unilus e pesquisa v.12, Revista Uningá v.56, Revista Liberum Accesum v.4, Revista acadêmica do instituto de ciências em saúde v.6, Revista Brazilian Journals of Health v.2, Journal of Aging Research v.07, Acta Biomédica Brasiliensia v.06, Artigos, Scielo.

Foram selecionados trabalhos entre os anos de 2015 a 2020. A coleta de dados ocorreu entre os meses de julho de 2020 a outubro 2021, utilizando os descritores: 'Atuação; fisioterapêutica'; 'Mal de Alzheimer'. Inicialmente, 25 trabalhos foram coletados, destes 18 se enquadraram ao tema. Ao utilizar o filtro dos últimos cinco anos (2015 a 2020), restaram 13 trabalhos que, após a realização da leitura, foram eletivos para compor este estudo. Os critérios de inclusão foram trabalhos relacionados ao tema e os objetivos propostos. Os critérios de exclusão foram trabalhos que não se encaixariam entre os anos de 2015 a 2020.

\section{REVISÃO DA LITERATURA}

\section{FISIOPATOLOGIA DO ALZHEIMER}

A patologia do Alzheimer é definida histopatologicamente como afecção neurodegenerativa progressiva associada ao envelhecimento e com alguns 
componentes genéticos, causada pela formação das placas senis e emaranhados neurofibrilares. (LIMA et al., 2019).

A formação das placas senis ocorre por um erro na clivagem da PPA, formando a proteína B-amilóide que se acumula no sistema nervoso, formando as placas senis que são tóxicas, rompendo, assim, as sinapses. Os emaranhados neurofibrilares se acumulam dentro da estrutura do neurônio devido a uma alteração da proteína tau, que tem função de estabilizar os microtúbulos, fazendo com que esta mude de formato e consequentemente perda sua função, formando, então, os emaranhados neurofibrilares, que causam degeneração do corpo celular. (SOARES, 2019).

Existem fatores de riscos que são associados ao aparecimento do mal de Alzheimer, dentre eles podemos citar: pessoas acima de 65 anos; sexo feminino; doenças cardiocirculatórias, como: hipertensão, acidente vascular encefálicos, dislipidemias, diabetes; alimentação deficiente em nutrientes e sedentarismo. (MEDEIROS et al., 2015).

Para o diagnóstico é necessário exame clínico, laboratorial, imagético e exclusão de outras demências. A doença de Alzheimer ainda não possui tratamento que possibilite a cura ou reversão das degenerações causadas, porém existem métodos farmacológicos e não farmacológicos que auxiliam na melhora da qualidade de vida do paciente. (MEDEIROS et al., 2015; SANTANA et al., 2019).

O tratamento não farmacológico inclui atuação de equipe multidisciplinar com prática diária de atividade física, estimulação cognitiva e fisioterapia, estas ações se mostram de grande importância para promoção, prevenção e recuperação. A fisioterapia possui meios que são utilizados com muita frequência na área da saúde afim de assistir aos idosos com mal de Alzheimer, buscando a conservação de funções cognitivas, motoras, retardando incapacidades funcionais e reabilitando o idoso funcionalmente. (SOARES, 2019; SILVA et al., 2019). 


\section{ATUAÇÃO FISIOTERAPÊUTICA}

Os exercícios fisioterapêuticos proporcionam uma melhor qualidade de vida aos que os praticam. A patologia do Alzheimer causa déficits importantes em seus portadores, desse modo a fisioterapia exerce papel fundamental na reabilitação desses pacientes. (MARINHO, 2020)

De modo geral os programas fisioterapêuticos administrados, nos estudos analisados em portadores da doença de Alzheimer, foram: a cinesioterapia, que se iniciada logo no início da patologia, prevenindo complicações cardíacas, articulares e do trato respiratório. Estas técnicas podem ser associadas à hidroterapia com exercício de carga, que proporcionam maior conforto e promovem o fortalecimento muscular. Os exercícios respiratórios, trazem como benefícios o aumento da capacidade pulmonar, fortalecimento da musculatura respiratória, reduzindo os riscos de complicações do trato respiratório, como a pneumonia, e evita acúmulo de secreções. Estes exercícios ajudam ainda, o idoso, no relaxamento e controle da ansiedade. (SOARES, 2019; LIMA et al., 2016)

Os exercícios aeróbicos previnem complicações cardíacas, neste caso, para pacientes que possuem a doença de Alzheimer, os estudos recomendam a prática de caminhada todos os dias. Este exercício possui baixo impacto e traz benefícios aos idosos, como: controle da pressão arterial, diminuição de dores articulares e prevenção de doenças como: osteoporose, artrite e artrose, sendo fundamental a presença de um acompanhante. (LIMA et al., 2016).

O fortalecimento muscular de membros superiores e inferiores reduzem o risco de quedas, que trazem como consequência as fraturas, sendo importante a atuação do fisioterapeuta para a promoção do fortalecimento, tendo em vista que as quedas são um acontecimento recorrente na vida dos idosos, que já possuem um desequilíbrio entre as células de absorção e regeneração na matriz óssea, osteoclastos e osteoblastos. Este desequilíbrio faz com que o osso se torne mais frágil, sendo a queda um indicativo de perda da funcionalidade, independência e causa de internações e morte entre os idosos. Portanto, exercícios de fortalecimento melhoram 
o equilíbrio e proporcionam aos pacientes com a doença de Alzheimer realizar tarefas, como por exemplo: atividades relacionadas a higiene pessoal, fazendo com que estes tenham maior independência funcional. (FERREIRA e SANTOS, 2020; SILVA et al., 2015).

Em estágios mais avançados, a doença de Alzheimer, causa perda da mobilidade, deixando o idoso acamado. Neste contexto, o fisioterapeuta pode atuar com exercícios ativos para ganho de amplitude de movimento (ADM), pois, estes são benéficos para alongar a musculatura, evitando encurtamentos musculares e preservando o movimento destes. (SANTOS; RODRIGUES; MONTEIRO, 2020)

Já os circuitos funcionais com obstáculos são utilizados para treino de marcha e reconquista do equilíbrio. As atividades que incluem jogos de memória com cores, palavras cruzadas, jogos de adivinhação e contagem das séries dos exercícios, quando colocados no programa de reabilitação do paciente com Alzheimer, associados à cinesioterapia, foi constatado ganhos cognitivos importantes. (FERREIRA e SANTOS, 2020)

Pacientes com doença de Alzheimer devem ser submetidos a uma avaliação global. A fisioterapia possui instrumentos que visam avaliar a independência funcional, como o índice de Barthel, que mede o grau de assistência exigida por uma pessoa, composto por 10 itens, envolve a mobilidade e os cuidados pessoais, e o miniexame do estado mental (MEEM), utilizado para avaliar a função cognitiva. O uso destes instrumentos se faz importante durante o tratamento fisioterapêutico, pois verificam a evolução do paciente diante do tratamento proposto. (MARINHO, 2020)

\section{CONSIDERAÇÕES FINAIS}

A fisioterapia e suas áreas de atuação se mostraram imprescindíveis na melhora da qualidade de vida de idosos com doença de Alzheimer, trazendo resultados satisfatórios, quando iniciada logo no início do descobrimento da patologia.

O presente artigo visou responder: Quais as condutas fisioterapêuticas descritas na literatura para os pacientes com doença de Alzheimer? Em resposta, verificou-se que 
as condutas fisioterapêuticas como: a cinesioterapia com exercícios de fortalecimento, alongamentos, exercícios respiratórios e os circuitos funcionais, trazem benefícios importantes aos portadores desta patologia.

Os exercícios respiratórios aumentam a expansibilidade torácica, melhoram as trocas gasosas, fazendo com que esses pacientes não desenvolvam doenças respiratórias graves que cause internação e intubação. Os circuitos são importantes para melhora da deambulação e do equilíbrio e, quando associados aos exercícios de fortalecimento e alongamento da musculatura, evitam à diminuição da fraqueza muscular, prevenindo a ocorrência de quedas e consequentemente de fraturas.

Portanto, concluímos que a fisioterapia atua na manutenção das capacidades funcionais, assim como, reduz e retarda complicações decorrentes da patologia, fazendo com que estes portadores possam ser independentes funcionalmente.

\section{REFERÊNCIAS}

BUSHATSKY, Angela; ALVES, Luciana Correia; DUARTE, Yeda Aparecida de Oliveira; LEBRÃO, Maria Lúcia. Fatores associados as alterações de equilíbrio em idosos residentes no município de São Paulo em 2006: Evidências do estudo de saúde, bem-estar e envelhecimento (SABE). Rev. Bras. Epidemiol. v. 21, n. 4, 2019. Disponível em: https://www.scielo.br/j/rbepid/a/TCNN6scPCSCNSGRS7V3whCt/?lang=pt. Acesso em: 13 jul. 2020.

FERREIRA, Lays da Silva Costa; SANTOS, Gabriela Lopes. Efeitos da fisioterapia na capacidade funcional e qualidade de vida de pacientes com doença de Alzheimer: Uma revisão da literatura. Revista acadêmica do instituto de ciências e saúde.

v. 3 ,

n. 2, 2017.

Disponível em https://revistas.unifan.edu.br/index.php/RevistalCS/article/view/346/276. Acesso em: 06 out. 2021.

LIMA, Andressa Maria Amorin de; SOUSA, Laís Botelho de; SOUZA, Maria Thanara Wanderley; SIQUEIRA, Thomas Décio Abdalla. O papel da fisioterapia no 
tratamento da doença de Alzheimer: Uma revisão da literatura. BIUS - Boletim Informativo Unimotrisaúde em Sociogerontologia v. 7, n. 1, 2016. Disponível em: https://www.periodicos.ufam.edu.br/index.php/BIUS/article/view/2610. Acesso em: 14 jul. 2020.

MANSO, Maria Elisa Gonzalez; SILVA, Deborah Lorenzo Gomez; OLIVEIRA, Rafaela Ramos d'; KIM, Beatriz Myung Jin; SANTOS, Ana Flavia de Lima; JUNIOR, Eduardo Antonio Barros Silva. Impacto do exercício físico em idosos com baixo peso: Uma abordagem focada na sarcopenia. PAJAR-Pan American Journal of Aging Research. v. $7, \quad$ n. 2, 2019. Disponível em: https://revistaseletronicas.pucrs.br/index.php/pajar/article/view/33308. Acesso em: 18 jul. 2020.

MARINHO, Matheus Falcão Santos. A importância da fisioterapia na doença de Alzheimer. Enveronmetal Smoke v. 3, n. 1, p. 69-78, 2020. Disponível em https://www.environmentalsmoke.com.br/index.php/EnvSmoke/article/view/85/74. Acesso em: 30 set. 2021.

MEDEIROS, Ingrid Maria Paes Jorge; SECURELLA, Fabiana Franco; SANTOS, Rita de Cássia Caramêz Saraiva; SILVA, Karina Martin Rodrigues. A influência da fisioterapia na cognição de idosos com doença de Alzheimer. Revista unilus e pesquisa. $\quad$ v. $12, \quad$ n. 29, 2015. Disponível em: http://revista.unilus.edu.br/index.php/ruep/article/view/686/u2015v12n29e686. Acesso em: 25 jul. 2020.

SANTANA, Carolina Manoela de Lima; NASCIMENTO, Larissa Gabriela Carvalho do; DANTAS, Suzanna dos Santos; VIEIRA, Thais Aline Evangelista; VIDAL, Giovanna Pontes; SOUZA, Adriana Paula Braz de. Capacidade funcional do paciente com Alheimer: Uma revisão integrativa. v. 19, n. 2, p. 271-285, 2019. Disponível em: http://temasemsaude.com/wp-content/uploads/2019/05/19215.pdf. Acesso em: 01 ago. 2020. 
SANTOS, Gisandra Cardoso dos; RODRIGUES, Gabriela Meira de Moura.; MONTEIRO, Eliane Maria de Oliveira. A influência da fisioterapia em pacientes com Alzheimer. Revista Liberum Accesum v. 4 p. 46-53, 2020. Disponível em: http://revista.liberumaccesum.com.br/index.php/RLA/issue/view/12. Acesso em: 04 out. 2021.

SILVA, Francisco Luís Cunha; SANTANA, Wilson Ribeiro de; RODRIGUES, Tatyanne Silva. Envelhecimento ativo: $O$ papel da fisioterapia na melhoria da qualidade de vida da pessoa idosa: Revisão integrativa. Revista uningá. v. 56, n. 4, p. 134-144, 2019. Disponível em: http://revista.uninga.br/index.php/uninga/article/view/2321/1952. Acesso em: 08 ago. 2020.

SILVA, Geraedson Aristides da; SANTOS, Clistenis Clênio Cavalcante dos; ALMEIDA, Carlos Daniel Fernandes de. Efeitos da cinesioterapia nos doentes de Alzheimer: Análise Bibliométrica. Acta Biomedica Brasiliensia v. 6, n. 1, 2015. Disponível em: https://www.actabiomedica.com.br/index.php/acta/article/view/101. Acesso em: 01 out. 2021.

SOARES, Reinaldo. Conduta fisioterapêutica na doença de Alzheimer, humanismo e ética. Braz. Rev. J.H. v. 2 n. 5, p. 4116-4123, 2019. Disponível em: https://www.brazilianjournals.com/index.php/BJHR/article/view/3386/3233. Acesso em: 16 ago. 2020.

SOUSA, Neuciani Ferreira da Silva; LIMA, Margareth Guimarães; CÉSAR, Chester Luiz Galvão; BARROS, Marlisia Berti de Azevedo. Envelhecimento Ativo: Prevalência e diferença de gênero e idade um estudo de base populacional. Cadernos de Saúde pública. n. 34, n. 11, 2018. Disponível em: https://www.scielo.br/pdf/csp/v34n11/1678-4464-csp-34-11-e00173317.pdf. Acesso em: 20 ago. 2020.

VERAS, Renato; OLIVEIRA, Martha. Envelhecer no Brasil: A construção de um modelo de cuidado. Ciência saúde coletiva. v. 23, n. 6, 2018. Disponível em: 
https://www.scielo.br/j/csc/a/snwTVYw5HkZyVc3MBmp3vdc/?format=pdf\&lang=pt. Acesso em 23 ago. 2020.

Enviado: Julho, 2021.

Aprovado: Dezembro, 2021. 